\title{
Flame Retardancy of Cellulose Fabrics Treated with 3-(Hydroxyphenyl Phosphinyl) Propanoic Acid
}

\author{
Lianping Zhang, Sam Soo Kim and Jaewoong Lee ${ }^{\dagger}$ \\ School of Textile, Yeungnam University, Gyeongsan, 712-749, Korea \\ ${ }^{1}$ Industrial Materials Research Institute, Kolon Industries, Inc., Gumi, 730-030, Korea
}

(Received: August 25, 2008/Revised: September 9, 2008/Accepted: October 10, 2008)

\begin{abstract}
Hydroxyphenyl phosphinyl) propanoic acid (HPPA) has been one of the most commonly used durable flame retardant agents for polyethylene terephthalate (PET) for many years. We intended to explore the application of HPPA to cellulose fabrics as formaldehyde-free phosphorus based flame retardants (FRs) through green chemistry process. The flame retardancy of the flame-retardant treated cellulose fabrics were characterized by using inductively coupled plasma spectroscopy (ICP) and limiting oxygen index (LOI). Structural changes of the treated cellulose fabrics were carried out by thermogravimetric analysis (TGA) and Fourier transform infrared (FT-IR) spectroscopy. To enhance the flame retardancy of HPPA treated cellulose fibers, glycerol polyglycidyl ether (GPE), a crosslinking agent was employed. Both HPPA and GPE treated cotton fabric imparted an LOI value over 26.
\end{abstract}

Keywords: flame retardant, cellulose, crosslinking agent, cotton, coupling agent, limiting oxygen index

\section{Introduction}

Phosphorus based flame retardants (FRs) have wide spectrum of advantages and are of interest for industrial and public area related to polymers and fibers, due to non-toxic emission during burning unlike some halogen-containing FRs ${ }^{1)}$. However, for cellulose fibers, the conventional phosphorus based FR, $N$-methylol dimethylphosphonopropionamide (MDPA) is not free from formaldehyde. In addition, during the treatment of MDPA on cellulose fibers, relatively excess amount of phosphoric acid is employed as a catalyst and alkali including sodium hydroxide could be used to neutralize the fabric which contains the phosphoric acid. Thus, the treatment of MDPA on cellulose fibers could produce toxic wastes.

In case of formaldehyde-free durable press finishes, polycarboxylic acids have been studied as substitutes for $\mathrm{N}$-methyol reagents. Especially, 1,2,3,4-butanetetracarboxylic acid (BTCA), a tetra-carboxylic acid, resulted in prominent durable press finishes ${ }^{2-6}$. Succinic acid
(SUA), a di-carboxylic acid also esterifies cellulose through an anhydride intermediate mechanism ${ }^{2,5)}$.

3-(Hydroxyphenyl phosphinyl) propanoic acid (HPPA, Fig. 1), one of popular phosphorus-based FRs, has two reactive groups in the structure which are carboxylic acid and phosphinic acid. In industrial applications, HPPA is mainly used to manufacture FR-PET. During synthesis of FR-PET, HPPA is connected among PET chains. The reaction of HPPA in producing FR PET resembles terephthalic acid (TPA) ${ }^{7-9)}$.

Through the review of literature, no trials to apply HPPA onto cellulose polymers for FR property were found. We intend to study the application of HPPA onto cellulose for the possibility of HPPA to form an anhydride intermediate during heating similarly with SUA, di-carboxylic acid. The anhydride form of HPPA would react with hydroxyl groups of cellulose through ring opening reaction and result in FRcellulose, which is a formaldehyde-free and leaving less toxic waste. In this research, the reactivity of HPPA with cellulose fibers and the flame retardancy 
<smiles>O=C(O)CCP(=O)(O)c1ccccc1</smiles>

Fig. 1. Molecular structure of HPPA.

of the HPPA-treated cellulose fiber will be discussed. In addition, the proposed reaction mechanism between HPPA and cellulose fiber will be suggested.

\section{Experimental}

\subsection{Materials}

Bleached cotton (KS K 0905) fabric was purchased from Korea Apparel Testing \& Research Institute. 3-(Hydroxyphenyl phosphinyl) propanoic acid (HPPA) under the commercial name of "HIRETAR-205" and glycerol polyglycidyl ether (GPE) as a cross-linking agent were kindly supplied by Kolon Industries, Inc..

\subsection{Fabric treatment}

Cotton fabrics were treated with the FRs formations of different concentrations in ethanol/water $(50 / 50, \mathrm{v} / \mathrm{v})$ solution, and were padded through a labboratory padder. The treated fabrics were dried at 85 for $5 \mathrm{~min}$, followed by curing at various temperatures for $3 \mathrm{~min}$. The cured fabrics were soaked in ethanol/distilled water $(50 / 50, \mathrm{v} / \mathrm{v})$ solution for $10 \mathrm{~min}$ to remove unreacted chemicals, followed by rinsing with distilled water.

\subsection{Dyeing with reactive dyes}

The HPPA-treated fabrics were dyed with C. I. Reactive Blue 4 and C. I. Reactive Blue 19 using an IR dyeing machine (KS L-24 PERFECT, Korean Science CO., LTD). The dyeing method is shown in Fig. 2, and the structures of the dyes are given in Fig. 3.

\subsection{Measurements}

$\mathrm{K} / \mathrm{S}$ values were investigated by using an $\mathrm{X}$-Rite Match-Rite spectrophotometer coupled to a PC using illuminant $\mathrm{D}_{65}$ and $10^{\circ}$ standard observer with the specular component excluded and the UV component included. Each fabric was folded once so as to give twice the thickness and an average of four readings was taken each time.

$$
\mathrm{K} / \mathrm{S}=(1-\mathrm{R})^{2} / 2 \mathrm{R}
$$

where, $\mathrm{K}$ is an absorption coefficient of the dyed samples, $S$ is a scattering coefficient $\lambda$ of the dyed samples and $\mathrm{R}$ is a spectral reflectance at max.

Limiting oxygen index (LOI) values of the fabrics were measured according to the GB/T standard method 5454-1997. An YG-813 oxygen index tester (Changzhou No.2 Textile Machinery Co., LTD) was used to determine the volumes of $\mathrm{O}_{2}$ and $\mathrm{N}_{2}$ required for ignition and the onset of burning. LOI values were calculated according to the following equation.
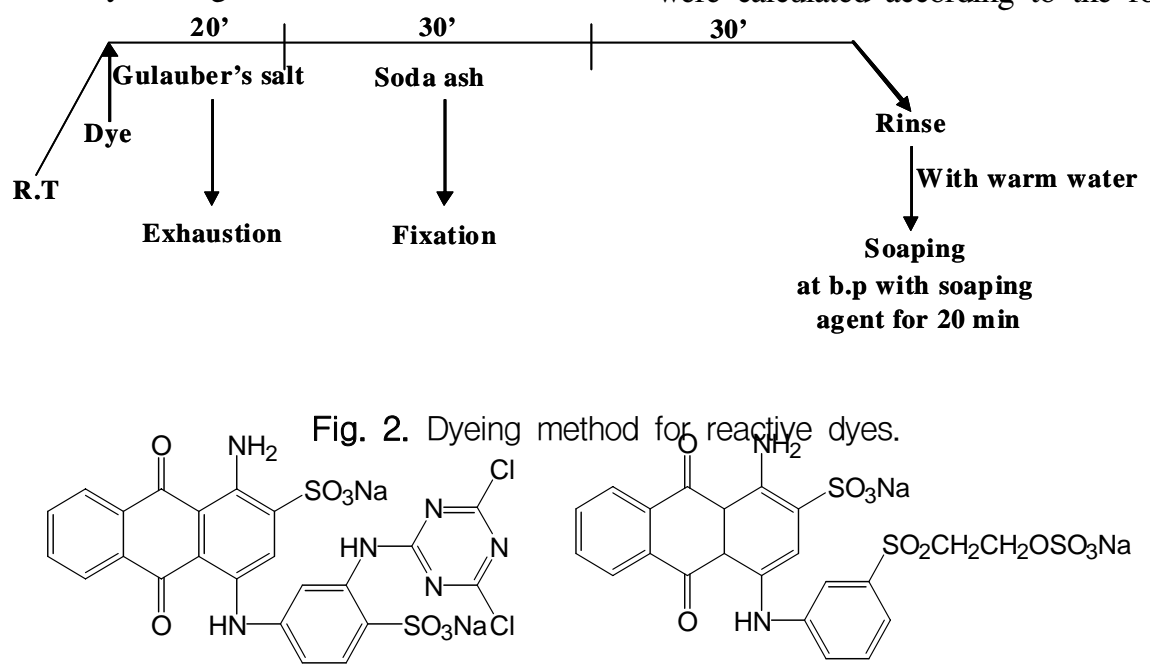

(a)

(b)

Fig. 3. Structures of the reactive dyes:

| 2 | www.ksdf.or.kr

(a) C. I. Reactive Blue 4, (b) C. I. Reactive Blue 19. 


$$
\mathrm{LOI}(\%)=\left[\mathrm{O}_{2}\right] /\left[\mathrm{O}_{2}\right]+\left[\mathrm{N}_{2}\right]
$$

where, $\left[\mathrm{O}_{2}\right]$ and $\left[\mathrm{N}_{2}\right]$ are the minimum oxygen and nitrogen volume concentration in the inflow gases respectively, required to pass the "minimum burning length" criterion.

Phosphorus concentration was characterized by using inductively coupled plasma spectroscopy (ICP) (Optima 5300DV, Perkin Elmer). Thermogravimetric analysis (TGA) were carried out under nitrogen atmosphere at a heating rate of $10 / \mathrm{min}$ using a TGA-50 thermal analyzer (DuPont, U.S.A.). The FT-IR spectra were recorded with a Spectrum GX (Bruker Co., Germany). Trace analysis was conducted by scanning electron microscopy (SEM) (S-4200, Hitachi Co., Japan).

\section{Results and discussion}

\subsection{Reaction of HPPA with cellulose}

In general, some polycarboxylic acids have been employed to form crosslink among cellulose chains for wrinkle-free finishing ${ }^{2-6}$. At an elevated temperature, polycarboxylic acids form anhydride intermediates followed by ring opening reaction with hydroxyl groups of cellulose. HPPA also would provide an anhydride intermediate with a five membered ring at an increased temperature during continuous finishing. In fact, HPPA has two reactive groups (Fig. 1), i.e. carboxylic acid and phosphinic acid which are similar to di-carboxylic acids, specifically, SUA. Therefore, HPPA may esterif an anhydride intermediate on cellulose. To prove the reactivity between HPPA and cellulose fibers, FT-IR spectra were obtained. In Fig. 4, in the untreated cotton fabric, there is no carbonyl band. However, the HPPA-treated cotton fabric contains the ester carbonyl band at $1722 \mathrm{~cm}^{-1}$ which occur between HPPA and cellulose. The carbonyl band of carboxylic acid in HPPA appeared at $1730 \mathrm{~cm}^{-1}$. Thus, it is presumed that the esterification between HPPA and cellulose occurred through an anhydride intermediate (Fig. 5).

In fact, the second carboxylic acid of SUA left after esterification does not react to cellulose due to difficulty to form a cyclic anhydride intermediate ${ }^{2,5}$.

A different method to prove the reaction between HPPA and cellulose fabrics is also conducted. After chemical bonding of cellulose fabrics with HPPA, there are reduced hydroxyl groups on cellulose left. It could be possible that reactable materials with hydroxyl groups of cellulose are employed to measure the active hydroxyl groups on cellulose. Due to excellent reactivity with hydroxyl groups on cellulose, reactive dyes were used. The strength of color of reactive dyes on cellulose fiber after dyeing is readily measured by $\mathrm{K} / \mathrm{S}$ value.

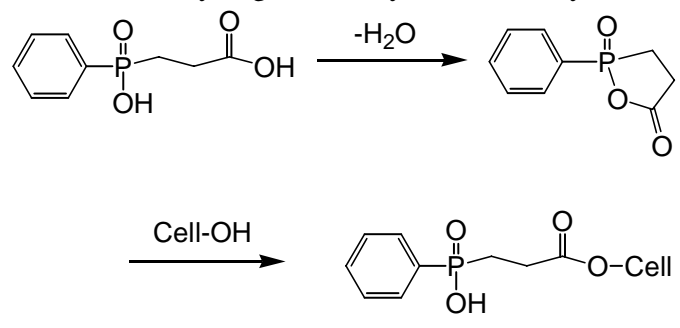

Fig. 5. Proposed reaction mechanism of HPPA with cellulose.

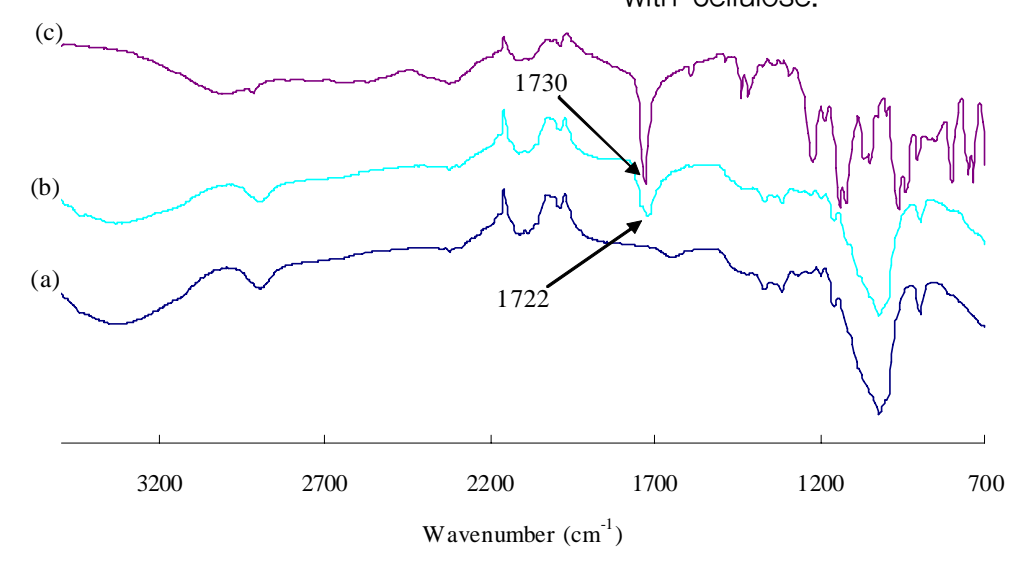

Fig. 4. FT-IR spectra of (a) untreated cotton (b) cotton treated with HPPA and (c) HPPA. 


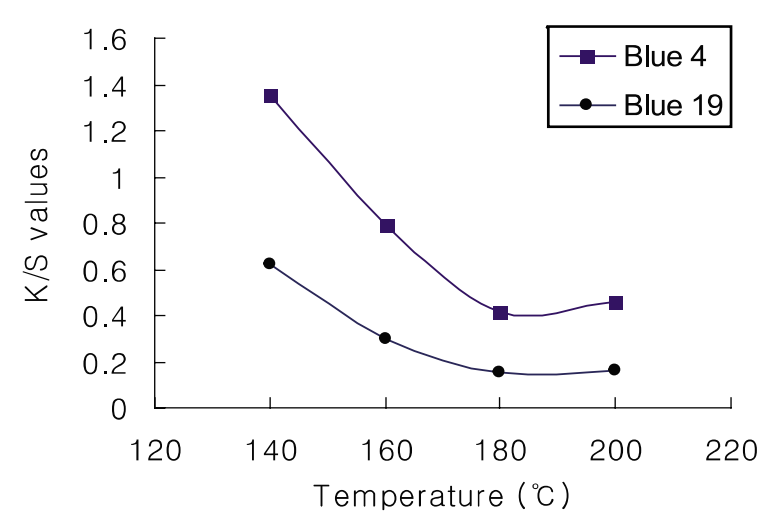

Fig. 6. The effect of curing temperature of HPPApadded cotton fabrics on the $\mathrm{K} / \mathrm{S}$ values in the subsequent dyeing with C. I. Reactive Blue 4 and C. I. Reactive Blue 19.

The higher $\mathrm{K} / \mathrm{S}$ value, the more reactive dyes are reacted to the hydroxyl groups. Fig. 6 shows that increased curing temperatures result in lower $\mathrm{K} / \mathrm{S}$ values. Therefore, the lower color strength of the HPPA-treated cotton fabrics suggested that many hydroxyl groups of cellulose fibers were reacted with HPPA. For two kinds of reactive dyes of C. I. Reactive Blue 4 (Procion M, Anthraquinone Dichlorotriazinyl) and Blue 19 (Procion H Anthraquinone Vinylsulphonyl), simitar results were obtained. In addition, at 180 and 200 the $\mathrm{K} / \mathrm{S}$ values of the treated fabrics showed similar intensity. It is assumed that the reactivity between cellitiose and HPPA could have a critical point around 180 a curing time of $3 \mathrm{~min}$. The proposed reaction mechanism of HPPA with cellulose fabrics is shown in Fig. 5.

\subsection{The flame retardancy of treated cotton}

Another method to measure the reactivity of HPPA to cellulose is to characterize the phosphorus content after the curing process. Fig. 7 gives the change of the phosphorus content on the treated cotton fabrics at different curing temperatures.

The maximum amount of phosphorus, $5467 \mathrm{ppm}$, was obtained under 180 and 3 min curing condition, which also indicates that the reaction between HPPA and cellulose fabrics occurred sufficiently.

Limiting Oxygen Index (LOI) value is known to determine flame retardancy of specific materials. The flame retardancy of the untreated and treated cellulose fibers is assessed by LOI measurements.

| 4 | www.ksdf.or.kr

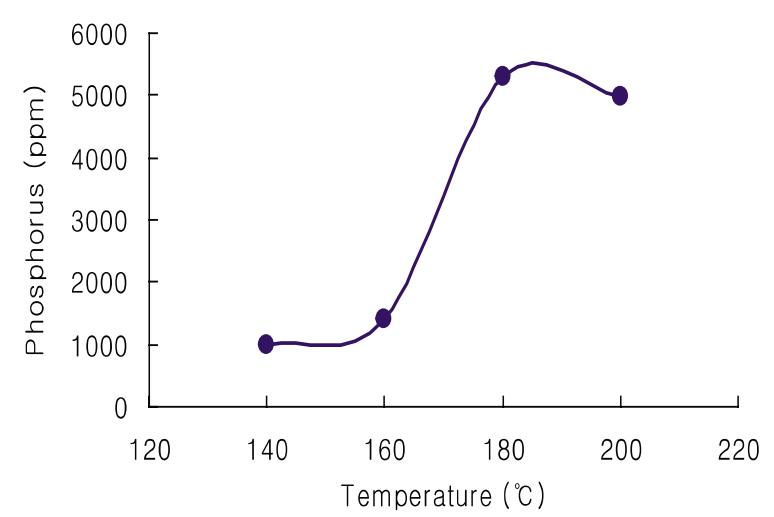

Fig. 7. Phosphorus content of the cotton fabrics treated with HPPA according to curing temperature.

Table 1. LOI values of the cellulose fabrics treated

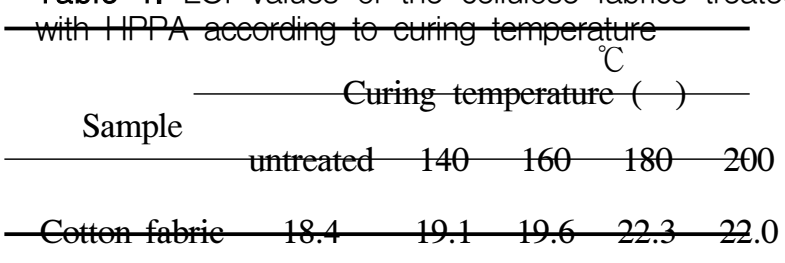

The results in Table 1 show that with increasing curing temperature, the LOI values are reached up to 22.3; however, in terms of flame retardancy, the LOI value of the HPPA treated cotton fabrics is not sufficient to be used as flame-retardant fabrics. Hence, to enhance flame retardancy of HPPA treated cellulose fibers, a different method was applied. In industrial area, crosslinking agents, especially, glycerol based epoxy resins are used as coupling agents for various adhesive applications. Among glycerol-based coupling agents, glycerol polyglycidyl ether (GPE) has two or three epoxy rings in the structure (Fig. 8), and the epoxy rings could react to carboxylic and phosphinic acid of HPPA through ring opening reaction. During the reaction between HPPA and cotton, in general, one hydroxyl group in cotton could connect to one HPPA after curing. However, in case of GPE, epoxy rings in GPE would provide secondary alcohols after connecting to hydroxyl group of cotton (Fig. 8).

These secondary alcohols or other epoxy rings left on GPE-cotton connection could react with HPPA. Thus, one hydroxyl group in cotton may be connected to more than one HPPA which may result in enhanced LOI values.

Table 2 indicates the improved flame retardancy of the cotton fabric treated with GPE and HPPA. Since 

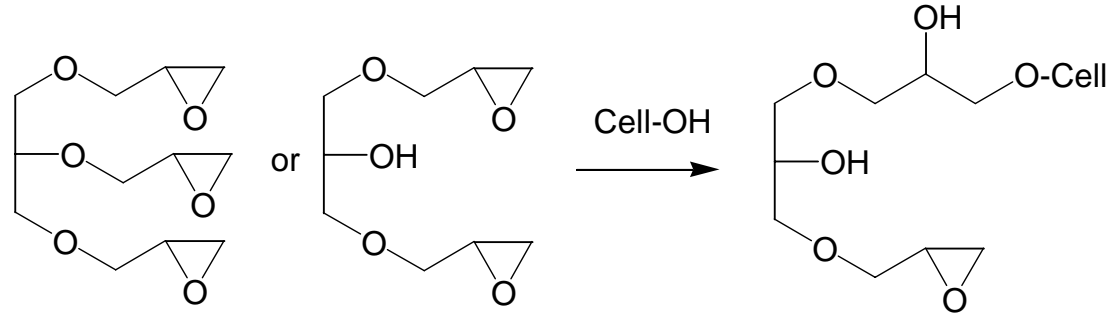

Fig. 8. Molecular structure of GPE and reaction scheme with cellulose.

Table 2. LOI values and phosphorous content of the

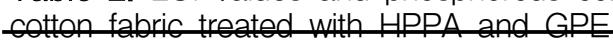

\begin{tabular}{ccc} 
Sample & LOI & $\begin{array}{c}\text { Phosphorous } \\
\text { concentration } \\
(\mathrm{ppm})\end{array}$ \\
\hline${ }^{\circ} \mathrm{C}$ & $15360{ }^{\circ} \mathrm{C}$ \\
\hline${ }^{\text {a Sample was dried at } 85}$ for 5 min and cured at 180
\end{tabular}

the sample with GPE possessed a LOI value over 26, the treated cotton shows proper flame retadancy. However, the LOI value of treated cotton fabrics with HPPA and GPE was not substantial.

\subsection{Thermal properties and morphology after burning of treated fabrics}

Fig. 9 shows the TGA curves of the cotton fabrics treated with HPPA. The untreated cotton ${ }^{\circ}$ fabric has a decomposition temperature starting at 340 whereas the HPPA-treated fabrics have lower decomposition temperatures.

It is assumed that a catalytic dehydration of cellulose by the phosphorus acid, which was provided from decomposition of the phosphorus-based FRs, resulting in decreased decomposition temperatures ${ }^{10,11)}$.

There was few residue/char left at 500 for the untreated cotton. In particular, the amount of char formed of the fabrics treated with both HPPA and GPE, is higher than the fabrics treated with HPPA only. Thus, it is assumed that the increased char formation might lead to improved flame retardancy of the treated fabrics. To ensure the effect of char left after burning, SEM was employed. Fig. 10 shows SEM images of chars formed from the untreated cotton and FR-treated cotton. The chars obtained from the untreated cotton were fuzzy; however, the chars of the FRtreated cotton were quite firm and intact. Most phosphorus-based FRs act by the condensed phase

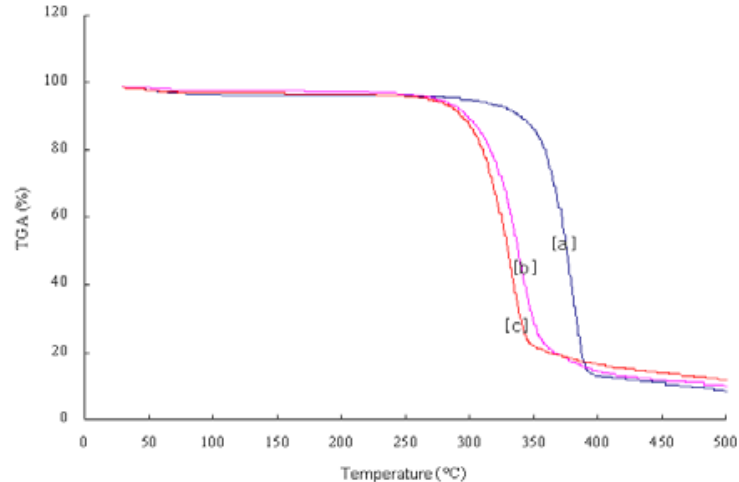

Fig. 9. Thermal stability of cotton fabrics: (a) untreated cotton, (b) treated with HPPA and (c) treated with HPPA and GPE.
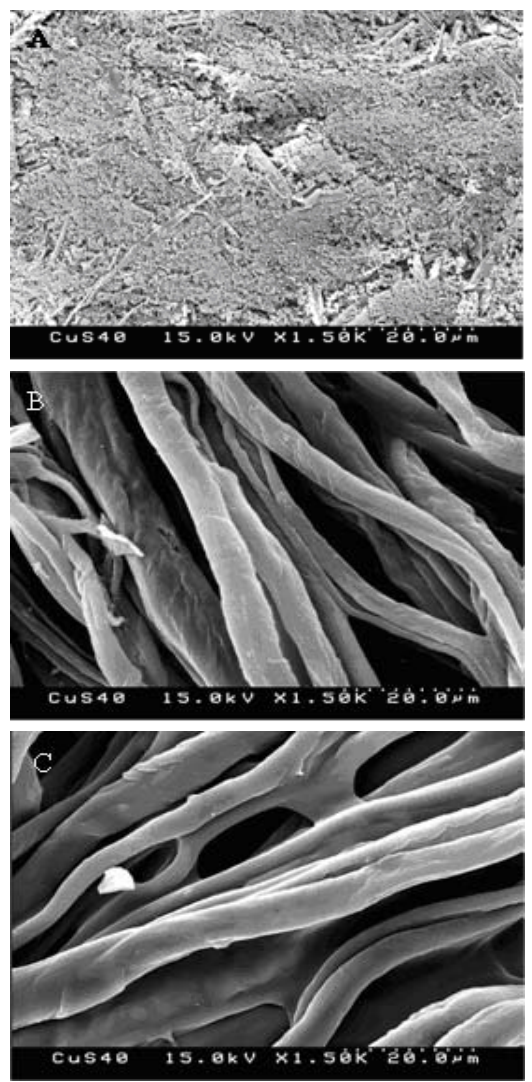

Fig. 10. SEM images of char left after burning: (a) untreated cotton, (b) cotton treated with HPPA and (c) cotton treated with HPPA and GPE.

J. of the Korean Soc. of Dyers and Finishers, Vol. 20, No. 5 | 5 | 
mechanism, during which the substrates dehydrate to form more char and protective coating of polyphosphoric acid, polyphosphoramidic structures or phosphorus oxynitride structures on the surface of the burning materials. In case of the FR-treated cotton, the integrity of fiber structures was retained.

This may be due to formation of polyphosphoric acid inside the fiber resulting in more crosslinked structures in the char. It resembles that the coating on fiber surfaces improves flame retardancy of the cellulose during the burning process ${ }^{12)}$.

\section{Conclusions}

This study suggests that HPPA, a conventional phosphorus FR for PET could be applied to cellulose fabrics and be a substitute for MDPA as a formaldehyde-free phosphorous-based FR.

HPPA would esterify cellulose via formation of an anhydride intermediate. After dyeing with reactive dyes, the decreased $\mathrm{K} / \mathrm{S}$ values of HPPA treated cotton fabrics along with the curing temperatures indicated increased esterification between HPPA and cellulose. By the co-use of a crosslinking agent, GPE, the LOI value of HPPA treated cotton fabrics was over 26 with $1.5 \%$ phosphorus concentration. Char structure of GPE/HPPA treated cotton results in relatively firm and intact form. Further research to enhance the LOI value of HPPA treated cotton fabrics is needed and phosphorus-nitrogen synergism using materials containing nitrogen would be considered.

\section{References}

1. S. V. Levchik and E. D. Weil, A Review of Recent Progress in Phosphorus-based Flame Retardants, J. Fire Sci., 24, 345-364(2006).

2. C. Q. Yang, X. Wang and I. Kang, Ester Crosslinking of Cotton Fabric by Polymeric Carboxylic Acids and Citric Acid, Textile Research Journal, 67(5), 334-342(1997).
3. C. Q. Yang, C. Hu and G. C. Lickfield, Crossliking Cotton with Poly(itaconic acid) and In Situ Polymerization of Itaconic Acid: Fabric Mechanical Strength Retention, J. Appl. Polym. Sci., 87, 20232030(2003).

4. C. Q. Yang and X. Wang, Infrared Spectroscopy Studies of the Cyclic Anhydride as the Intermediate for the Ester Crosslinking of Cotton Cellulose by Polycarboxylic Acids. II. Comparison of Different Polycarboxylic Acids, J. Polym. Sci., Part A: Polym. Chem., 34, 1573-1580(1996).

5. C. Q. Yang and X. Wang, Formation of Cyclic Anhydride Intermediates and Esterification of Cotton Cellulose by Multifunctional Carboxylic Acids: An Infrared Spectroscopy Study, Textile Research Journal, 66(9), 595-603(1996).

6. J. Lee, R. M. Broughton, A. Akdag, S. D. Worley and T. S. Huang, Antimicrobial Fibers Created via Polycarboxylic Acid Durable Press Finishing, Textile Research Journal, 77(8), 604-611(2007).

7. S. Yang and J. Kim, Flame Retardant Polyesters. I. Phosphorous Flame Retardants, J. Appl. Polym. Sci., 106, 2870-2874(2007).

8. S. Yang and J. Kim, Flame Retardant Polyesters. II. Polyester Polymers, J. Appl. Polym. Sci., 106, 1274-1280(2007).

9. S. Yang and J. Kim, Flame Retardant Polyesters. III. Fibers, J. Appl. Polym. Sci., 108, 2297-2300 (2007).

10. G. Joseph, "Fire Retardancy of Polymeric Materials", Marcel Dekker, Inc., New York, pp.148-206, 2000.

11. S. Gaan and G. Sun, Effect of Phosphorus Flame Retardants on Thermo-oxidative Decomposition of Cotton, Polym. Degrad. Stab., 92, 968-974(2007).

12. S. Gaan and G. Sun, Effect of Phosphorus and Nitrogen on Flame Retardant Cellulose: A Study of Phosphorus Compounds, J. Anal. Appl. Pyrolysis, 78, 371-377(2007). 